randomised clinical trials assessed the treatment-independent trends for overall colon cleansing quality versus overall colon lesion detection.

Methods Three multi-centre phase 3 clinical trials compared the cleansing efficacy and safety of the 1L PEG NER1006 $\left(\right.$ PLENVU $\left.^{\oplus}\right)$ versus standard bowel preparations in patients aged 18-85 years. Treatment-blinded assessment of colon cleansing quality was performed by both site colonoscopists (SC; who also detected all lesions per local practice) and by central readers (CR). Two validated cleansing scales were used: the Harefield Cleansing Scale (HCS) and the Boston Bowel Preparation Scale (BBPS; only by CR). Patients with documented HCS cleansing grades D-A, overall BBPS scores $0-9$, and overall colon lesion counts were included in this analysis. Logistic regression trends were fitted to polyp- (PDR) and adenoma (ADR) detection rates, using cleansing quality as a covariate.

Results Out of 1,985 randomised patients, 1,749 patients were included (table 1). With site colonoscopists' HCS grades, the logistic regression for relative lesion detection demonstrated an odds ratio of 1.17 , i.e. for each incremental increase in the HCS colon cleansing grade from $\mathrm{D}$ to $\mathrm{A}$ there was a 1.17 times increase in both PDR $(\mathrm{P}=0.009)$ and $\operatorname{ADR}(\mathrm{P}=0.019)$. With central readers, the corresponding increase was 1.24 times for PDR $(\mathrm{P}=0.005)$ and 1.26 times for ADR $(\mathrm{P}=0.006)$. With central readers, each incremental increase in the successful BBPS scores 6-9 resulted in a 1.08 times increase in PDR $(\mathrm{P}<0.001)$ and 1.10 times increase in $\operatorname{ADR}(\mathrm{P}<0.001)$ versus failing BBPS scores 0-5.

Conclusions Adopting a better bowel preparation remains a good way to improve quality in colonoscopy. With both HCS and BBPS, an increased overall colon cleansing quality was associated with greater overall colon PDR and ADR across the full range from cleansing failures and up to high-quality cleansing.

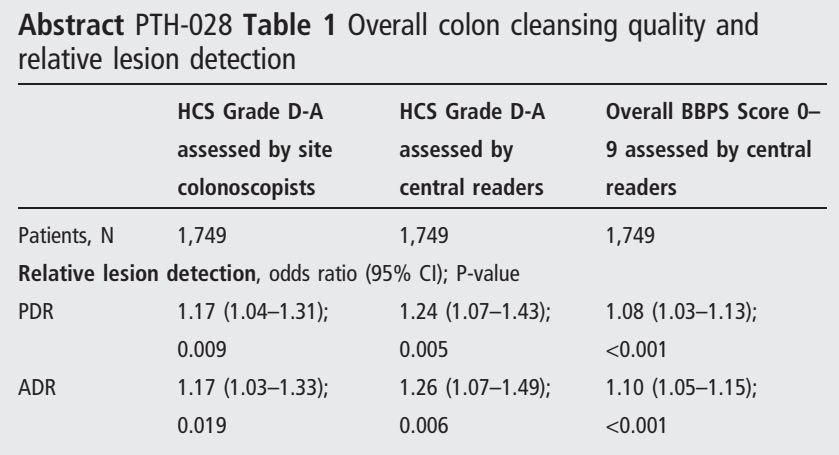

\section{PTH-029 CLOSURE OF GASTROCUTANEOUS FISTULAS WITH AN OVER-THE-SCOPE-CLIP (OTSC) - A LARGE TERTIARY HOSPITAL EXPERIENCE}

Thomas Hollingworth*, Philip Boger, Emily Clarke, Praful Patel, Charlotte Rutter, Trevor Smith, Imdadur Rahman. University Hospital Southampton, Southampton, UK

\subsection{6/gutinl-2019-BSGAbstracts.54}

Introduction Gastrocutaneous fistulae (GF) are a rare, but difficult to manage complication after percutaneous endoscopic gastrostomy (PEG) tube removal. In cases, refractory to conventional medical management various approaches have been used for their treatment including; surgical closure, through the scope clipping and endoscopic suturing with variable results. The over-the-scope-clip (OTSC) has an emerging role in the endoscopic closure of gastrointestinal wall defects, including persistent GF. The aim of this study was to assess the efficacy and safety of the use of OTSC for the closure of persistent GF.

Methods A prospectively kept database was analysedfrom September 2016 to January 2019 was undertaken of all persistent GF using an OTSC 12/6 GF clip. Prior to the deployment of an OTSC, the fistulous tract was disrupted using a wire brush, which also acted as a guide for placement. The use of ancillary techniques such as snare debulking or knife resection was utilised if needed. Primary outcome measures were; successful deployment of the OTSC, procedure time, complications and 30 day success. Secondary outcome measures were PEG dwell time prior to removal, time from diagnosis to closure and sedation type.

Results A total of 22 procedures were performed, on 12 male \& 10 female patients with a mean age of 47 . Median PEG dwell time was 16 months prior to removal. 64\% of the procedures were performed with conscious sedation, with $36 \%$ requiring enhanced sedation with anaesthetic support. Technical success was $100 \%$, with 2 procedures requiring ancillary techniques to facilitate the deployment of the OTSC, resulting in 1 complication of intra-procedural bleeding (stopped endoscopically). The median procedure time was 14 mins (range 9-35). A 30 \& 90 day success was reported in $96 \%$ cases, with only one fistula recurring.

Discussion This is the largest cohort of patients with a persistent GF treated with an OTSC. This evolving procedure is an effective and safe method for the treatment of persistent GF. It has a much lower morbidity compared to surgery and shorter procedure times than more invasive endoscopic treatment options, such as endoscopic suturing.

\section{PTH-030 OUTCOMES OF HEMOSPRAY USE IN PEPTIC ULCER UPPER GASTROINTESTINAL BLEEDS: OUTCOMES FROM THE HEMOSPRAY REGISTRY}

\begin{abstract}
${ }^{1,2}$ Mohamed Hussein*, ${ }^{2}$ Durayd Alzoubaidi, ${ }^{3}$ Miguel Fraile, ${ }^{3}$ Jacobo Ortiz Fernandez-sordo, ${ }^{3}$ Krish Ragunath, ${ }^{4}$ Radu Rusu, ${ }^{4}$ John Dunn, ${ }^{14}$ Johannes Rey, ${ }^{5}$ Shraddha Gulati, ${ }^{5}$ Bu'Hussain Hayee, ${ }^{6}$ Selena Dixon, ${ }^{6}$ Sulleman Moreea, ${ }^{7}$ Duncan Napier, ${ }^{7} J o h n$ Anderson, ${ }^{8}$ Martin Dahan, ${ }^{9}$ Max Hu, ${ }^{9}$ Patricia Duarte, ${ }^{9}$ Phil Boger, ${ }^{10}$ John Mcgoran, ${ }^{10}$ Inder Mainie, ${ }^{11}$ Alberto Murino, ${ }^{11}$ Sina Jameie- oskooei, ${ }^{11} \mathrm{Edward}$ Despott, ${ }^{12}$ Cora Steinheber, ${ }^{12}$ Martin Goetz, ${ }^{13}$ Sharmila Subramaniam, ${ }^{13}$ Pradeep Bhandari, ${ }^{1,2}$ Laurence Lovat, ${ }^{8}$ Emmanuel Coron, ${ }^{14}$ Ralf Keisslich, ${ }^{1,2}$ Rehan Haidry. ${ }^{1} U C L H, U K ;{ }^{2} U C L$, UK; ${ }^{3}$ Nottingham Digestive Diseases Centre, UK; ${ }^{4}$ Guys and St Thomas Hospitals, UK; ${ }^{5}$ Kings college Hospital, UK; ${ }^{6}$ Bradford Teaching Hospital NHS Trust, UK; ${ }^{7}$ Gloustershire Hospitals NHS Foundation Trust, UK; ${ }^{8}$ University Hospital of Nantes, France; ${ }^{9}$ University Hospital Southampton, UKi ${ }^{10}$ Belfast NHS Trust, UK; ${ }^{11}$ Royal Free Hospital, UK; ${ }^{12}$ Tübingen University Hospital,UK; ${ }^{13}$ University of Portsmouth, UK; ${ }^{14}$ Horst Schmidt Kliniken, Germany
\end{abstract}

\subsection{6/gutjnl-2019-BSGAbstracts.55}

Introduction Peptic ulcers are the commonest cause of upper Gastrointestinal bleeding (UGIB). Hemospray (Cook Medical, North Carolina, USA) is a novel haemostatic powder aimed to treat UGIB. The aim of this study is to look at outcomes in patients with peptic ulcer GI bleeds treated with hemospray in 13 centres.

Methods Data was prospectively collected on hemospray use in UGIBs in the UK, France and Germany (Jan'16-Sept'18). Hemospray was used for peptic ulcer UGIBs as a 
monotherapy, dual-therapy with standard haemostatic techniques or rescue therapy. Haemostasis was defined as cessation of bleeding within 5 minutes of hemospray application.

Results 196 patients with UGIBs secondary to peptic ulcers were recruited (133 M, 63 F, 123/196(63\%) duodenal, 44/ $196(22 \%)$ gastric, 29/196(15\%) oesophageal). Immediate haemostasis was achieved in $171 / 196(87 \%)$ patients. The median rockall score was 7 (IQR, 6-8).

Rebleeding rates were significantly lower in forrest $2 \mathrm{a}$ relative to current predicted rebleeding rates based on forrest classifications, $2 / 21(10 \%, \mathrm{P}<0.005)$. In the $25 / 196(13 \%)$ patients who did not achieve haemostasis 18/25 (72\%) were Forrest $1 \mathrm{~b}$ ulcers. In the total cohort, 33/169(20\%) had a rebleed, median rockall score was $7(\mathrm{IQR}, 7-8)$.

Outcomes with different Forrest classifications (table 1)

\begin{tabular}{|c|c|c|c|c|}
\hline \multicolumn{5}{|c|}{ Abstract PTH-030 Table 1} \\
\hline & Forrest 1 a $(n=37)$ & $\begin{array}{l}\text { Forrest } 1 b \\
(n=111)\end{array}$ & Forrest $2 a(n=23)$ & $\begin{array}{l}\text { Forrest } 2 b \\
(n=20)\end{array}$ \\
\hline \multirow[t]{2}{*}{ Haemostasis } & $31 / 37$ & 93/111 & $23 / 23(100 \%)$ & $19 / 20$ \\
\hline & $(84 \%)$ & $(84 \%)$ & & $(95 \%)$ \\
\hline \multirow[t]{2}{*}{ Median Blatchford } & 13 & 12 & 12 & 12 \\
\hline & IQR: $11-14$ & IQR:9-15 & IQR: 11-14 & IQR: $11-15$ \\
\hline \multirow[t]{2}{*}{ Median Rockall } & 7 & 7 & 7 & 7 \\
\hline & IQR: 6-8 & IQR:6-8 & IQR: 6-7 & IQR: 6-8 \\
\hline \multicolumn{5}{|c|}{ Rockall score 7 predicted re-bleeding rate: $25-40 \%$} \\
\hline \multirow[t]{2}{*}{ Re-bleeding } & $8 / 31$ & 19/94 & $2 / 21(10 \%)$ & $4 / 18$ \\
\hline & $(26 \%)$ & $(20 \%)$ & & $(22 \%)$ \\
\hline \multicolumn{5}{|c|}{ Rockall score 7 predicted mortality: $20-30 \%$} \\
\hline \multirow[t]{2}{*}{ 30-day mortality } & $9 / 31$ & $21 / 94$ & $3 / 21(14 \%)$ & $5 / 18$ \\
\hline & $(29 \%)$ & $(22 \%)$ & & $(28 \%)$ \\
\hline
\end{tabular}

Conclusions Hemospray is effective in achieving immediate haemostasis in peptic ulcer UGIBs. The baseline Blatchford/ rockall scores in our cohort are high with patients recruited from tertiary centres with high-risk cases. The rebleeding and mortality rates are in keeping/below the predicted rate based on the scores. The best outcome with hemospray was with forrest $2 \mathrm{a}$ ulcers.

\section{PTH-031 IS FLEXIBLE SIGMOIDOSCOPY IN THE SETTING OF NORMAL COMPUTER TOMOGRAPHIC COLONOGRAPHY NECESSARY?}

Harleen Johal*, Karen Hartery, Vikrant Kale, Vincent Cheung, Ioannis Koutsounis, Colin Ferrett, Adam Bailey. John Radcliffe Hospital, Oxford, UK

\subsection{6/gutjnl-2019-BSGAbstracts.56}

Introduction The demand to deliver rapid diagnosis of colorectal cancer (CRC) safely and effectively continues to increase. The practice of combining flexible sigmoidoscopy (FS) and CT colonography (CTC) has been driven by a concern that CTC misses some rectosigmoid cancers. NICE guidelines state that CTC can be considered as an alternative to colonoscopy for diagnosis, if a radiology service can demonstrate competency in this technique. The Oxford University Hospitals Foundation Trust OUHFT two-week wait (2WW) referral pathway for suspected CRC states that patients over 75 years, or over 60 years with chronic medical co-morbidities and symptoms suspicious of CRC should have both FS and CTC (with low dose prep for frail patients). The aim of this audit was to assess the additional cancer yield of FS in the setting of normal CTC.

Methods Analysis of all referrals $(n=941)$ meeting the above criteria over a thirteen-month period (July 2015 - August 2016) was performed. Of these referrals, 851 CTCs were reviewed and 901 FSs. Endoscopic data was obtained from reports, including findings, bowel preparation, study quality, level reached, reasons for failure and histopathology. Radiological data was obtained from CTC reports, taking into account the findings, technique, study quality and recommendations. The two data sets were then directly compared, with particular focus on the correlation between CTC and FS when identifying left-sided CRC.

Results 37 L-sided CRCs were identified on FS. The splenic flexure was only reached in $22.1 \%(n=200)$ of the studies, with poor bowel preparation being the most frequently documented reason for failure. 87 possible CRCs were identified on CTC. Of these, 34 were right-sided CRCs, 9 were leftsided suspicious polyps, and 44 were left-sided CRCs. When correlating the radiology, histopathology and endoscopic findings, there were $7 \mathrm{CRCs}$ identified on CTC that were not seen on FS. Of these, 4 were histologically proven to be CRCs that were missed due to incomplete endoscopic procedure. By comparison, only one $20 \mathrm{~mm}$ polyp, a lateral spreading tumour (LST) seen on FS was missed on CTC. 447 CTCs $(52.5 \%)$ were reported as showing benign colonic disease (e.g. diverticular disease, small polyps) and further endoscopic evaluation was advised in 27.7\% $(\mathrm{n}=124)$.

Conclusion We demonstrated that all CRCs seen on FS were identified on CTC. In the setting of normal CTC, only 1 LST was missed. By amending the OUHFT $2 \mathrm{WW}$ referral pathway, so that CTC is completed first, with subsequent endoscopic evaluation only if the CTC is inconclusive or the reporting radiologist recommends direct visualisation, we anticipate substantial resource saving without compromising diagnostic performance for colorectal cancer.

\section{PTH-032 A STUDY OF THE YIELD OF INVESTIGATION IN RECURRENT IRON DEFICIENCY ANAEMIA}

${ }^{1}$ Christopher John* ${ }^{2}$ Nicola Whiller, ${ }^{2}$ Carla Smith, ${ }^{2}$ Jonathan Snook, ${ }^{2}$ Lachlan Ayres. ${ }^{1}$ Portsmouth Hospitals Nhs Foundation Trust, UK; ${ }^{2}$ Poole Hospital NHS Foundation Trust

\subsection{6/gutjnl-2019-BSGAbstracts.57}

Introduction Recurrent iron deficiency anaemia (IDA) is a relatively common clinical condition, with potentially serious gastro-intestinal (GI) causes. Previous studies have shown that 9.6-25\% of patients with negative initial upper and lower GI investigations go on to develop recurrent IDA, with up to $55 \%$ of these patients being diagnosed with a GI pathology. ${ }^{1}$ 2 There are well established guidelines on initial investigation, but there are few data on the yield of reinvestigation for recurrent IDA. The aim of this study was to determine the diagnostic yield from investigating recurrent IDA, using a larger dataset than the few previous smaller studies.

Methods The electronic patient records of the prospectively maintained IDA database at Poole Hospital NHS Foundation Trust were retrospectively examined. Patients were categorised as recurrent IDA if they re-presented a year or more after their initial investigation. Data were collected on index and 